\title{
DETERMINATION OF THE THREE-DIMENSIONAL VELOCITY FIELD IN A GLACIER*
}

\author{
By C. F. Raymond $\dagger$ \\ (California Institute of Technology, Pasadena, California 9ırog, U.S.A.)
}

\begin{abstract}
A method for the determination of the three-dimensional velocity field in a glacier is described. Measurements in three or more bore holes arranged in an appropriate array are needed for its application. Surface motion measured by triangulation and tape measure, bore-hole profiles given by inclinometry, and the geometry of the bed are all considered simultaneously in order to determine the velocity field. The basic assumption is that velocity between the bore holes can be represented by suitable interpolation based on the measurements in the holes. Ice displacement parallel to bore holes is calculated indirectly from incompressibility and the constraint that velocity normal to the bed be zero. As an example, the method is applied to an array of 9 bore holes in Athabasca Glacier.

RÉsumé. Détermination du champ des vitesses dans les trois dimensions sur un glacier. On décrit une méthode pour la détermination du champ des vitesses dans les trois dimensions sur un glacier. Dans son application il faut faire des mesures dans 3 ou plus trous de forage répartis suivant une disposition appropriée. Les mouvements en surface mesurés par triangulation et chainage, les profils des forages donnés par inclinométrie et la géométrie du lit sont pris en compte simultanément afin de déterminer le champ de vitesse. L'hypothèse de base est que la vitesse entre les forages peut être représentée par une interpolation convenable basée sur les mesures dans les forages. Le déplacement de la glace parallèlement aux forages est calculé indirectement à partir de l'incompressibilité de la glace et de l'obligation que la vitesse normale au lit soit pulle. La méthode est appliquée, à titre d'example, pour un reseau de 9 trous de forage dans le Glacier de l'Athabasca.

Zusammenfassung. Bestimmung des dreidimensionalen Geschwindigkeitsfeldes in einem Gletscher. Eine Methode zur Bestimmung des dreidimensionalen Geschwindigkeitsfeldes in einem Gletscher wird beschrieben. Zu ihrer Anwendung sind Messungen in drei oder mehr geeignet angeordneten Bohrlöchern notwendig. Zur Bestimmung des Geschwindigkeitsfeldes werden gleichzeitig die Oberflächenbewegung, gemessen mittels Triangulation und Messband, Bohrlochprofile, bestimmt mit Hilfe der Klinometrie, und die Geometrie des Gletscherbettes herausgezogen. Die grundlegende Annahme dabei ist, dass die Geschwindigkeit zwischen den Bohrlöchern durch geeignete Interpolation, die sich auf die Messungen in den Löchern stützt, erfasst werden kann. Die Eisverschiebung parallel zu den Bohrlöchern wird indirekt aus der Inkompressibilität und der Zwangsbedingung, dass die Geschwindigkeit senkrecht zum Untergrund gleich Null ist, berechnet. Als Beispiel wird die Methode auf eine Anordnung von 9 Bohrlöchern auf dem Athabasca Glacier angewendet.
\end{abstract}

\section{INTRODUGTION}

Measurement of tilting in isolated single bore holes cannot give complete strain-rate information at depth in a glacier. The analysis of such measurements therefore requires some assumptions about the pattern of flow at depth (Gerrard and others, 1952; Nye, I957; Savage and Paterson, r963). Although such assumptions can often be supported by indirect observations and theoretical arguments, tests of the natural rheological behavior of glacier ice and the applicability of theoretical analyses of glacier flow become more convincing with a direct measurement of all of the strain-rate components. Tilt measurements in several bore holes arranged in a suitable array make it possible to determine the components of velocity and strain-rate within a glacier with a significantly reduced number of assumptions. The purpose of this paper is to describe a general technique for completely determining internal velocity and strain-rate from measurements in three or more holes. It is applied to measurements made in a nine-hole array on Athabasca Glacier.

The problem of determining ice velocity and deformation from bore-hole measurements needs special consideration because of a fundamental deficiency in the techniques presently used. Tilt measurements within a bore hole and location of the surface intersection of a bore hole by triangulation can define the coordinates of points on the hole trace, but are not sufficient to distinguish different ice elements along the hole. Thus the motion of the bore hole

* Publication 1928 of the Division of Geological Sciences, California Institute of Technology.

$\dagger$ Now at University of Washington, Seattle, Washington 98105, U.S.A. 
as determined by such measurements repeated in time can only give the components of velocity normal to the hole trace, but not the component parallel to the trace. One must assume a distribution of velocity parallel to the hole in order to compute the three components of velocity in any coordinate system of interest. Even if a technique were devised for tagging elements of ice along the length of the hole, measurements in a single hole can at best determine the hole-parallel gradients of velocity or three independent velocity gradients. For an unambiguous determination of the components of strain-rate, knowledge of all nine velocity gradients is required. The purpose of a bore-hole array is to allow the estimation of some of the remaining velocity gradients by comparison of displacements in adjacent holes as done by Shreve and Sharp (1970), Savage and Paterson (I963), and Kamb and Shreve (I966).

After introducing necessary notation, formulae relating measured displacement in a single bore hole to the components of velocity and strain-rate are derived. Then it is shown how these formulae can be used in conjunction with a continuity condition (incompressibility) and a suitable scheme for interpolating between bore holes to calculate self-consistent velocity and strain-rate fields for the volume of ice included within the bore-hole array.

\section{CoORdinates AND NOTATION}

The method is described using an orthogonal right-handed coordinate system $(x, y, z)$ such that the $y=0$ plane approximates the glacier surface in the area of the bore-hole array and the $y$ axis points downward. For the purpose of the following description the $x$ and $z$ axes can assume any fixed azimuthal orientation. The $x$ and $z$ coordinates of a hole at time $t$ are denoted as $x_{\mathrm{h}}(y, t)$ and $z_{\mathrm{h}}(y, t)$. The two functions $x_{\mathrm{h}}(y, t), z_{\mathrm{h}}(y, t)$ give a parametric representation of the hole trace at time $t$. $x$ and $z$ components of tilt are defined as

$$
\gamma_{x}(y, t)=\frac{\partial x_{\mathbf{h}}(y, t)}{\partial y}, \quad \gamma_{z}(y, t)=\frac{\partial z_{\mathrm{h}}(y, t)}{\partial y} .
$$

For the purpose of the analysis it is assumed that the functions $x_{\mathbf{h}}(y, t), z_{\mathbf{h}}(y, t)$ have been determined by surface triangulation and integration of measured bore-hole tilts at two distinct times $t_{\mathrm{i}}$ and $t_{\mathrm{f}}$ separated by an interval $\Delta t$. For convenience of notation, hole coordinates and tilt components at times $t_{\mathrm{i}}$ and $t_{\mathrm{f}}$ are denoted by superscripted quantities, e.g. $x_{\mathrm{h}}\left(y, t_{\mathrm{i}}\right)=x_{\mathrm{h}}{ }^{\mathrm{i}}(y)$, etc.

\section{SiNGLE BORE-HOLE ANALYSIS}

Since a bore hole is approximately vertical on initial implacement, it is sub-parallel to the $y$ axis when the glacier surface is not very steep as is usually the case. Thus to a first approximation the components of velocity normal to the bore hole are a direct measure of the $x$ and $z$ components of velocity $u$ and $w$. With this in mind formulae are developed which give $u$ and $w$ and their gradients in the $y$ direction in terms of the measured bore-hole displacements and additional terms of smaller contribution. These terms involve knowledge of the third component of velocity $v$ and the $x$ and $z$ gradients of all three components of velocity along the bore hole and are of increasing importance where the glacier surface is steep and the pattern of flow deviates significantly from simple shear parallel to the surface.

The basic equations. Consider the bore-hole displacement in the $x y$ plane (Fig. I). Since the $y$ component of velocity $v$ is in general non-zero, a specified element of ice initially at depth $y^{\mathrm{i}}$ lies at a different depth $y^{\mathrm{f}}$ after the time interval $\Delta t$. The average $y$ velocity of the ice element is $\left(y^{\mathfrak{f}}-y^{\mathrm{i}}\right) / \Delta t$. The path of the element projected on the $x y$ plane is some curve joining $x_{\mathrm{h}}^{\mathrm{i}}\left(\nu^{\mathrm{i}}\right)$ and $x_{\mathrm{h}}^{\mathrm{P}}\left(y^{\mathrm{P}}\right)$ as indicated by the dashed curve in Figure $\mathrm{I}$. The average $x$ component of velocity of the element during the interval $\Delta t$ is then $\left[x_{\mathrm{h}}^{\mathrm{f}}\left(y^{\mathrm{f}}\right)-x_{\mathrm{h}}{ }^{\mathrm{i}}\left(y^{\mathrm{i}}\right)\right] / \Delta t$. 


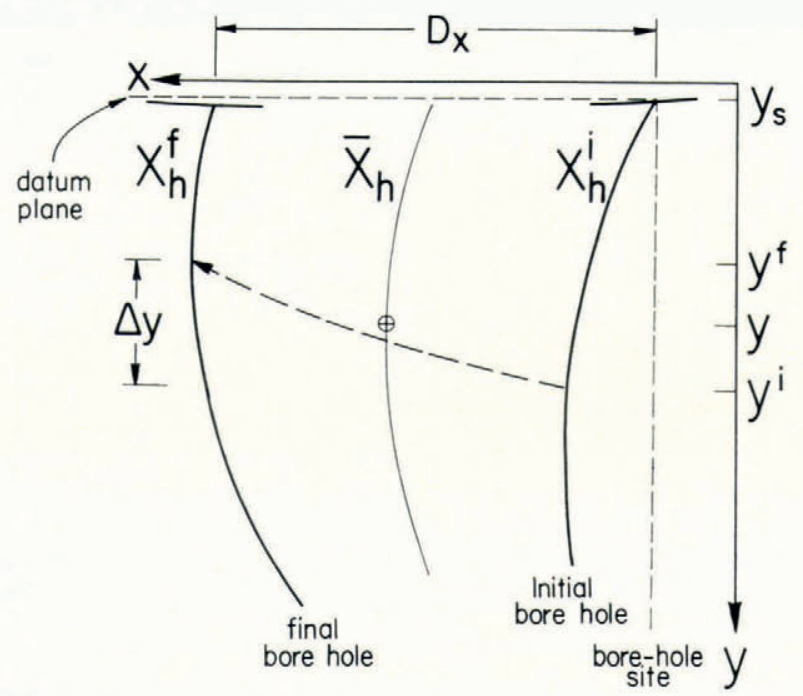

Fig. I. View of hole displacement projected on xy plane.

The average velocity of an ice element is related to the velocity at a specific point in space as follows. Let $\Delta y=y^{\mathrm{f}}-y^{\mathrm{i}}$, and consider a depth $y=\left(y^{\mathrm{f}}+y^{\mathrm{i}}\right) / 2$ halfway between the initial and final depths of the ice element. Define

$$
\bar{v}(y)=\frac{\mathrm{I}}{\Delta t}\left(y^{\mathrm{f}}-y^{\mathrm{i}}\right)=\frac{\Delta y}{\Delta t}
$$

and

$$
\bar{u}(y)=\frac{\mathrm{I}}{\Delta t}\left[x_{\mathrm{h}}{ }^{\mathrm{f}}\left(y+\frac{1}{2} \Delta y\right)-x_{\mathrm{h}}^{\mathrm{i}}\left(y-\frac{1}{2} \Delta y\right)\right] .
$$

$\bar{u}(y)$ and $\bar{v}(y)$ represent the average velocity in the $x y$ plane of the bore-hole element for which $y^{\mathrm{i}}$ and $y^{\mathrm{f}}$ are equal distances above and below depth $y$. Now define

$$
\begin{aligned}
& \bar{x}_{\mathrm{h}}(y)=\frac{1}{2}\left[x_{\mathrm{h}^{\mathrm{f}}}(y)+x_{\mathrm{h}^{\mathrm{i}}}(y)\right], \\
& \bar{z}_{\mathrm{h}}(y)=\frac{1}{2}\left[z_{\mathrm{h}^{\mathrm{f}}}(y)+z_{\mathrm{h}^{\mathrm{i}}}(v)\right] .
\end{aligned}
$$

$\bar{x}_{\mathrm{h}}(y)$ and $\bar{z}_{\mathrm{h}}(y)$ define a curve which is half-way between the initial and final hole traces. Intuitively it could be expected that the velocity of an ice element averaged over its path would be about the same as the velocity at a point half-way between its initial and final positions. This point is indicated by the circled cross in Figure I. Thus it is assumed that

$$
\begin{aligned}
& \bar{v}(y) \approx v\left(\bar{x}_{\mathrm{h}}(y), y, \bar{z}_{\mathrm{h}}(y)\right), \\
& \bar{u}(y) \approx u\left(\bar{x}_{\mathrm{h}}(y), y, \bar{z}_{\mathrm{h}}(y)\right) .
\end{aligned}
$$

If elements of ice along the bore hole are tagged in order to give direct measurement of $\Delta y$, then $\bar{v}(y)$ and $\bar{u}(y)$ can be calculated from Equations (I) and (2). If such tagging is not done, then one must assume or estimate by other indirect measurements a distribution of $v$. This enables one to arrive at a value of $\bar{v}(y)$ from Equation (4) and $\Delta y$ from Equation (I) in order to calculate $\bar{u}(y)$ from Equation (2) and $u\left(\bar{x}_{\mathrm{h}}(y), y, \bar{z}_{\mathrm{h}}(y)\right)$ from Equation (5). The succeeding analysis is carried out for the latter case in which there is no direct measurement of $\Delta y$.

Before discussing in general the nature of the approximations involved in Equations (4) and $(5)$, it is worthwhile to consider them for simplified cases, which can be compared to 
other treatments of bore-hole data. For notational convenience in these discussions, the variables in Equations (6) to (12) are written without arguments. It should be held in mind that these equations involve flow quantities along the curve defined by Equations (3).

Consider the case for which the initial and final hole configurations are determined at times separated by only an infinitesimal time interval. In the limit $\Delta t \rightarrow 0$

$$
\begin{aligned}
& x_{\mathrm{h}}{ }^{\mathrm{P}}(y)=\bar{x}_{\mathrm{h}}(y)=x_{\mathrm{h}}{ }^{\mathrm{i}}(y)=x_{\mathrm{h}}\left(y, t_{\mathrm{i}}\right), \\
& z_{\mathrm{h}^{\mathrm{f}}}(y)=\bar{z}_{\mathrm{h}}(y)=z_{\mathrm{h}}{ }^{\mathrm{i}}(y)=z_{\mathrm{h}}\left(y, t_{\mathrm{i}}\right),
\end{aligned}
$$

and Equations (4) and (5) are exact. Equation (5) in combination with (1) (2) and (4) gives

$$
u=\frac{\partial x_{\mathrm{h}}}{\partial t}+\gamma x v
$$

This is equivalent to an equation given by Savage and Paterson (1963, p. 4525), when the finite time interval in their equation is made infinitesimal.

The differential form of Equation (6) can be derived either by direct differentiation of Equation (6) or by differentiation of Equations (2) and (5) and elimination of $\mathrm{d} \tilde{u} / \mathrm{d} y$. The origin of the various terms is best seen by following the latter route. Differentiation of Equation (5) gives

$$
\frac{\mathrm{d} \bar{u}}{\mathrm{~d} y}=\frac{\partial u}{\partial y}+\gamma_{x} \frac{\partial u}{\partial x}+\gamma_{z} \frac{\partial u}{\partial z} .
$$

This is identical with suitable change in notation, to an equation derived by Shreve and Sharp (1970, p. 78). Differentiation of Equation (2) gives

$$
\frac{\mathrm{d} \bar{u}}{\mathrm{~d} y}=\frac{\partial \gamma_{x}}{\partial t}+\frac{\partial \gamma x}{\partial y} v+\gamma_{x}\left(\frac{\partial v}{\partial y}+\gamma_{x} \frac{\partial v}{\partial x}+\gamma_{z} \frac{\partial v}{\partial z}\right)
$$

where Equations (I) and (4) have been used to express $\Delta y$ and its derivative with respect to $y$ in terms of $v$ and its gradients. Eliminating $\mathrm{d} u / \mathrm{d} y$ between Equations (7) and (8) gives

$$
\frac{\partial u}{\partial y}=\frac{\partial \gamma_{x}}{\partial t}+\frac{\partial \gamma_{x}}{\partial y} v-\gamma_{x}\left(\frac{\partial u}{\partial x}-\frac{\partial v}{\partial y}\right)-\gamma_{z} \frac{\partial v}{\partial z}+\gamma_{x}\left(\gamma_{x} \frac{\partial v}{\partial x}+\gamma_{z} \frac{\partial v}{\partial z}\right) \text {. }
$$

With the simplification under the assumptions that the hole is straight $\left(\partial \gamma_{x} / \partial y=0\right)$, the deformation field is of plane strain type $(\partial / \partial z=0)$, and the ice is incompressible $(\partial u / \partial x+\partial v / \partial y=0)$, one gets

$$
\frac{\partial u}{\partial y}=\frac{\partial \gamma_{x}}{\partial t}-2 \gamma_{x} \frac{\partial u}{\partial x} .
$$

This is identical except for differences in notation, to the formula given by Nye (1957, p. I30) in his analysis of the Jungfraufirn bore-hole experiment (Gerrard and others, I952). The above discussions show that the present treatment is equivalent to past analysis for an infinitesimal time interval.

Equations (4) and (5) are applicable when the time interval between the initial and final determination of the hole coordinates is finite. However, in this case they are only approximate. To investigate the nature of the approximation it is useful to derive an equation equivalent to Equation (ro) but without the assumption of an infinitesimal time interval. As before, Equations (2) and (5) are differentiated with respect to $y$ and combined to eliminate $\mathrm{d} \bar{u} / \mathrm{d} y$. Now, with the same assumptions about the bore hole and deformation field which lead to Equation (Io) and the additional assumption that the velocity gradients are homogeneous, one gets

$$
\frac{\partial u}{\partial y} \approx \frac{\mathrm{I}}{\Delta t}\left(\gamma x^{\mathrm{f}}-\gamma x^{\mathrm{i}}\right)-\left(\gamma x^{\mathrm{f}}+\gamma x^{\mathrm{i}}\right) \frac{\partial u}{\partial x} .
$$


This can be compared with Nye's equation for time-independent velocity (1957, p. I30)

$$
\frac{\partial u}{\partial y}=\frac{2 \partial u / \partial x}{\exp (2 \Delta t \partial u / \partial x)-\mathrm{I}}\left[\gamma x^{\mathrm{f}}-\gamma x^{\mathrm{i}} \exp \left(2 \Delta t \frac{\partial u}{\partial x}\right)\right],
$$

which is exact for a finite time interval under the above assumptions. This equation was derived by integration with respect to time of Equation (10). Expansion of Equation (12a) valid for $2 \Delta t \partial u / \partial x<2 \pi$ gives

$$
\left.\frac{\partial u}{\partial y}=\frac{\mathrm{I}}{\Delta t}\left(\gamma_{x^{\mathrm{f}}}^{\mathrm{f}}-\gamma x^{\mathrm{i}}\right)\left(\mathrm{I}+\frac{1}{3}\left(\Delta t \frac{\partial u}{\partial x}\right)^{2}\right)+O\left[\left(\Delta t \frac{\partial u}{\partial x}\right)^{4}\right]\right)-\left(\gamma x^{\mathrm{f}}+\gamma x^{\mathrm{i}}\right) \frac{\partial u}{\partial x}
$$

where $O\left[\left(\Delta t \frac{\partial u}{\partial x}\right)^{n}\right]$ represents the lowest order term with non-zero coefficient not explicitly written. Comparison of this last result with Equation (I I) shows that in this simple case, Equation (5) gives a good approximation when $\Delta t \partial u / \partial x$ is small compared to one.

More generally, if the velocity field is assumed only to be independent of time, it can be shown that

$$
\begin{gathered}
\Delta y=v\left(\bar{x}_{\mathrm{h}}(y), y, \bar{z}_{\mathrm{h}}(y)\right) \Delta t\left\{\mathrm{I}+O\left[\left(\Delta t \frac{\partial v_{j}}{\partial x_{k}}\right)^{2}\right]+O\left[\left(\Delta t^{2} v_{l} \frac{\partial^{2} v_{j}}{\partial x_{l} \partial x_{k}}\right)\right]\right\}, \\
\bar{u}(y)=u\left(\bar{x}_{\mathrm{h}}(y), y, \bar{z}_{\mathrm{h}}(y)\right)\left\{\mathrm{I}+O\left[\left(\Delta t \frac{\partial v_{j}}{\partial x_{k}}\right)^{2}\right]+O\left[\left(\Delta t^{2} v_{l} \frac{\partial^{2} v_{j}}{\partial x_{l} \partial x_{k}}\right)\right]\right\}, \\
\frac{\mathrm{d} \bar{u}(y)}{\mathrm{d} y}-\bar{\gamma}_{x}(y) \frac{\partial u}{\partial x}\left(\bar{x}_{\mathrm{h}}(y), y, \bar{z}_{\mathrm{h}}(y)\right)-\bar{\gamma}_{z}(y) \frac{\partial u}{\partial z}\left(\bar{x}_{\mathrm{h}}(y), y, \bar{z}_{\mathrm{h}}(y)\right) \\
=\frac{\partial u}{\partial y}\left(\bar{x}_{\mathrm{h}}(y), y, \bar{z}_{\mathrm{h}}(y)\right)\left\{\mathrm{I}+O\left[\left(\Delta t \frac{\partial v_{j}}{\partial x_{k}}\right)^{2}\right]+O\left[\left(\Delta t^{2} v_{l} \frac{\partial^{2} v_{j}}{\partial x_{l} \partial x_{k}}\right)\right]\right\},
\end{gathered}
$$

where the lowest-order terms in the first and second spatial derivatives of the components of velocity are indicated. The actual form of the higher-order terms for the general case is considerably more complicated than for the case represented by Equations (12). However, error terms with $\Delta t \partial v_{j} / \partial x_{k}$ raised to the first power still do not appear. By assigning the average velocity of an ice element as given by Equations (I) and (2) to a point closely halfway between its initial and final positions, an extra order of accuracy has been gained, as could be intuitively expected.

Modifications of the basic equations. Equations (I), (2), (4), and (5) permit one to calculate the distribution of $u$ along the curve defined by Equation (3) when $v$ is known on that curve. However, for purposes of interpretation it is often more desirable to know the velocity along a straight line normal to the surface, which in the present coordinates would be a line of constant $x$ and $z$. In addition, for purposes of calculating $x$ and $z$ gradients of velocity from velocity computed at adjacent bore holes, it is highly desirable to have the velocities given along lines of constant $x$ and $z$, so that the differentiation formulae are independent of depth. For these reasons the equations are modified so that only the values of flow quantities along the $y$ coordinate line which passes through the surface intersection of the initial bore-hole appear. For convenience in later discussion, this $y$ coordinate line is referred to as the borehole site. This is in contrast with the actual bore-hole location which depends on time and depth. If $y_{\mathrm{s}}$ represents the $y$ coordinate of the top of the initial hole trace as shown in Figure $\mathrm{I}$, then $x_{\mathrm{h}}{ }^{\mathrm{i}}\left(y_{\mathrm{s}}\right)$ and $z_{\mathrm{h}}{ }^{\mathrm{i}}\left(y_{\mathrm{s}}\right)$ give the $x$ and $z$ coordinates of the bore-hole site. To first order in $\left[\bar{x}_{\mathrm{h}}(y)-x_{\mathrm{h}}{ }^{\mathrm{i}}\left(y_{\mathrm{s}}\right)\right]$ and $\left(\bar{z}_{\mathrm{h}}(y)-z_{\mathrm{h}}{ }^{\mathrm{i}}\left(y_{\mathrm{s}}\right)\right]$, Equations (I) to (5) give:

$$
\Delta y \approx \Delta t\left\{v(y)+\frac{\partial v}{\partial x}(y)\left[\bar{x}_{\mathrm{h}}(y)-x_{\mathrm{h}}{ }^{\mathrm{i}}\left(y_{\mathrm{s}}\right)\right]+\frac{\partial v}{\partial z}(y)\left[\bar{z}_{\mathrm{h}}(y)-z_{\mathrm{h}}{ }^{\mathrm{i}}\left(y_{\mathrm{s}}\right)\right]\right\},
$$




$$
\begin{aligned}
u(y) \approx \frac{\mathrm{I}}{\Delta t}\left[x_{\mathrm{h}}{ }^{\mathrm{f}}\left(y+\frac{1}{2} \Delta y\right)-\right. & \left.x_{\mathrm{h}} \mathrm{i}\left(y-\frac{1}{2} \Delta y\right)\right]+ \\
& +\frac{\partial u}{\partial x}(y)\left[\bar{x}_{\mathrm{h}}(y)-x_{\mathrm{h}}{ }^{\mathrm{i}}\left(y_{\mathrm{s}}\right)\right]+\frac{\partial u}{\partial z}(y)\left[\bar{z}_{\mathrm{h}}(y)+z_{\mathrm{h}}{ }^{\mathrm{i}}\left(y_{\mathrm{s}}\right)\right] .
\end{aligned}
$$

For notational convenience velocity and velocity gradients are written simply as functions of $y$, but represent the values at points $\left(x_{\mathrm{h}}^{\mathrm{i}}\left(y_{\mathrm{s}}\right), y, z_{\mathrm{h}}{ }^{\mathrm{i}}\left(y_{\mathrm{s}}\right)\right)$. Equations (13) and (14) give $u$ at the bore-hole site in terms of $v$ and the $x$ and $z$ gradients of all three velocity components evaluated at the bore-hole site. No further approximation is involved if the $x$ and $z$ gradients of velocity are independent of $x$ and $z$.

One further useful modification of the analysis is to partition Equation (14) into contributions from the value of $u$ at the surface and the differential motion between the surface and a point at depth. Call the plane $y=y_{\mathrm{s}}$ the datum plane and let

$$
D_{x}=x_{\mathrm{h}}{ }^{\mathrm{f}}\left(y_{\mathrm{s}}\right)-x_{\mathrm{h}}{ }^{\mathrm{i}}\left(y_{\mathrm{s}}\right), \quad D_{z}=z_{\mathrm{h}^{\mathrm{f}}}\left(y_{\mathrm{s}}\right)-z_{\mathrm{h}}{ }^{\mathrm{i}}\left(y_{\mathrm{s}}\right)
$$

represent the hole displacements on the datum plane during the interval $\Delta t$ (Fig. I). Equation (14) applied at $y=y_{\mathrm{s}}$ gives

$$
u_{\mathrm{s}}=u\left(y_{\mathrm{s}}\right)=\frac{\mathrm{I}}{\Delta t} D_{x}-\frac{\mathrm{I}}{2} \frac{\partial u}{\partial x}\left(y_{\mathrm{s}}\right) D_{x}-\frac{\mathrm{I}}{2} \frac{\partial u}{\partial z}\left(y_{\mathrm{s}}\right) D_{z}+\frac{v\left(y_{\mathrm{s}}\right)}{2}\left[\gamma x^{\mathrm{f}}\left(y_{\mathrm{s}}\right)+\gamma x^{\mathrm{i}}\left(y_{\mathrm{s}}\right)\right]
$$

when the surface segment of the hole lacks curvature, which in practice is the case to a very good approximation. (Note that Equation (I5) also is applicable for calculation of surface velocity from measurements made on stakes frozen into the ice. It gives the velocity at the initial location of the stake.) Now let the hole coordinates be represented as

$$
\begin{aligned}
& x_{\mathrm{h}}(y, t)=x_{\mathrm{h}}\left(y_{\mathrm{s}}, t\right)+\Delta x_{\mathrm{h}}(y, t), \\
& z_{\mathrm{h}}(y, t)=z_{\mathrm{h}}\left(y_{\mathrm{s}}, t\right)+\Delta z_{\mathrm{h}}(y, t) .
\end{aligned}
$$

If $u(y)$ at depth is represented by

$$
u(y)=u\left(y_{\mathrm{s}}\right)+u_{\mathrm{d}}(y)
$$

then Equations (14) and (15) give

$$
\begin{aligned}
& u_{\mathrm{d}}(y) \approx \frac{\mathrm{I}}{\Delta t}\left[\Delta x_{\mathrm{h}}{ }^{\mathrm{f}}\left(y+\frac{1}{2} \Delta y\right)-\Delta x_{\mathrm{h}}{ }^{\mathrm{i}}\left(y-\frac{1}{2} \Delta y\right)\right]- \\
& \quad-\frac{\mathrm{I}}{2} \frac{\partial u}{\partial x}(y)\left[\Delta x_{\mathrm{h}}{ }^{\mathrm{f}}(y)+\Delta x_{\mathrm{h}}{ }^{\mathrm{i}}(y)\right]-\frac{\mathrm{I}}{2} \frac{\partial u}{\partial z}(y)\left[\Delta z_{\mathrm{h}}{ }^{\mathrm{f}}(y)-\Delta z_{\mathrm{h}}{ }^{\mathrm{i}}(y)\right]- \\
& \quad-\frac{\mathrm{I}}{2} \frac{\partial u_{\mathrm{d}}}{\partial x}(y) D_{x}-\frac{\mathrm{I}}{2} \frac{\partial u_{\mathrm{d}}}{\partial z}(y) D_{z}-\frac{v\left(y_{\mathrm{s}}\right)}{2}\left[\gamma x^{\mathrm{f}}\left(y_{\mathrm{s}}\right)+\gamma x^{\mathrm{i}}\left(y_{\mathrm{s}}\right)\right] .
\end{aligned}
$$

With

$$
w(y)=w\left(y_{\mathrm{s}}\right)+w_{\mathbf{d}}(y)
$$

identical considerations applied to the $x z$ plane give

$$
\begin{aligned}
& w_{\mathrm{d}}(y) \approx \frac{\mathrm{I}}{\Delta t}\left[\Delta z_{\mathrm{h}}{ }^{\mathrm{P}}\left(y+\frac{1}{2} \Delta y\right)-\Delta z_{\mathrm{h}}{ }^{\mathrm{i}}\left(y-\frac{1}{2} \Delta y\right)\right]- \\
& -\frac{\mathrm{I}}{2} \frac{\partial w}{\partial x}(y)\left[\Delta x_{\mathrm{h}}^{\mathrm{f}}(y)+\Delta x_{\mathrm{h}}^{\mathrm{i}}(y)\right]-\frac{\mathrm{I}}{2} \frac{\partial w}{\partial z}(y)\left[\Delta z_{\mathrm{h}^{\mathrm{f}}}(y)-\Delta z_{\mathrm{h}^{\mathrm{i}}}(y)\right]- \\
& -\frac{\mathrm{I}}{2} \frac{\partial w_{\mathrm{d}}}{\partial x}(y) D_{x}-\frac{\mathrm{I}}{2} \frac{\partial w_{\mathrm{d}}}{\partial z}(y) D_{z}-\frac{v\left(y_{\mathrm{s}}\right)}{2}\left[\gamma_{z^{\mathrm{P}}}\left(y_{\mathrm{s}}\right)+\gamma z^{\mathrm{i}}\left(y_{\mathrm{s}}\right)\right] .
\end{aligned}
$$

The greatest advantage of this seemingly more complex approach is that it provides an input channel for extra measurements at the surface, thereby improving to some extent the results at all depths. Surface velocity gradients (e.g. $\partial u\left(y_{\mathrm{s}}\right) / \partial x, \partial w\left(y_{\mathrm{s}}\right) / \partial z$, etc.) can be 
determined by triangulation and taping measurements in a wider network of stakes. Uncertainty in the $x$ and $z$ gradients resulting from a deficiency in number or spacing of bore holes can then be restricted to the calculation with respect to $u_{\mathrm{d}}$ and $w_{\mathrm{d}}\left(\mathrm{e} . \mathrm{g} . \partial u_{\mathrm{d}}(y) / \partial x\right.$, $\partial w_{\mathrm{d}}(y) / \partial z$, etc.).

An additional advantage of practical importance is that as long as the velocity field can be assumed to be time-independent, surface measurements and tilt measurements within the bore hole need not be made over the same time interval.

Indirect estimation of $v$. To complete the analysis for a single bore hole, it is only necessary to arrive at a distribution of $v$ versus $y$ at the bore-hole site. This decision must be based on the information at hand in each specific application. One possible way of proceding is as follows. When the surface layers of a glacier are not composed of firn as in the ablation zone, the ice can be assumed to be incompressible to a good approximation; thus,

$$
\frac{\partial u}{\partial x}+\frac{\partial v}{\partial y}+\frac{\partial w}{\partial z}=0
$$

In this case,

$$
v^{\star}(y)=v\left(y_{\mathrm{s}}\right)-\int_{y_{s}}^{y}\left(\frac{\partial u}{\partial x}\left(y^{\prime}\right)+\frac{\partial w}{\partial z}\left(y^{\prime}\right)\right) \mathrm{d} y^{\prime}
$$

would give a good approximation to $v(y)$. If the geometry of the glacier bed is known, additional input can be achieved by using the requirement that the velocity normal to the bed be zero as done by Savage and Paterson (1963). The value $v_{\mathrm{b}}$ that $v$ at the bed, depth $y_{\mathrm{b}}$, must have is

$$
v_{\mathrm{b}}=v\left(y_{\mathrm{b}}\right)=\beta_{x} u_{\mathrm{b}}+\beta_{z} w_{\mathrm{b}}
$$

where $\beta_{x}$ and $\beta_{z}$ are the $x$ and $z$ components of slope of the bed with respect to the $x z$ plane. Thus in order to take account of possible failure of the assumption of incompressibility and inaccuracy in $\partial u / \partial x$ and $\partial w / \partial z, v^{\star}$ can be adjusted to get an improved estimate of $v$ :

$$
v(y)=v^{\star}(y)-\left(v^{\star}\left(y_{\mathrm{b}}\right)-v_{\mathrm{b}}\right) \frac{\left(y-y_{\mathrm{s}}\right)^{2}}{\left(y_{\mathrm{b}}-y_{\mathrm{s}}\right)^{2}} .
$$

By using the square of the ratio $\left(y-y_{\mathrm{s}}\right) /\left(y_{\mathrm{b}}-y_{\mathrm{s}}\right)$, significant adjustment of $v^{\star}$ is limited to a zone close to the bed, where the uncertainty in evaluating $\partial u / \partial x$ and $\partial w / \partial z$ is greatest. Equation (I9) can also be partitioned into the value of $v$ at the surface and the value relative to the surface, so that

$$
v(y)=v\left(y_{\mathrm{s}}\right)+v_{\mathrm{d}}(y) .
$$

\section{ANALYSIS OF AN ARRAY OF BORE HOLES}

In order to apply the equations of the previous section to calculate the components of velocity at the site of a bore hole, the $x$ and $z$ gradients of velocity need to be known. Calculation of the $x$ and $z$ gradients of velocity requires knowledge of the velocity in several bore holes in a suitable array. This circular dilemma is conveniently overcome by the iterative procedure schematically illustrated in Figure 2. As preparation for the iterative cycle values of the components of velocity and their $x$ and $z$ gradients at the surface of the bore-hole sites are calculated from triangulation and tape measurements on surface markers and the surface intersection of the bore holes. The surface values of flow quantities are not changed in the iterative cycle. In the first step of the cycle, Equation (I 7), (I9), or some other equation is used to estimate $v(y)$. Equations ( $\mathrm{I} 6$ ) are used for the second step to get $u_{\mathrm{d}}$ and $w_{\mathrm{d}}$. In the third and final step interpolating functions are fitted at each depth to the values of $u_{\mathrm{d}}, v_{\mathrm{d}}$, and $w_{\mathrm{d}}$ calculated at the bore-hole sites. These functions represent the distribution of $u_{\mathrm{d}}$, 
$v_{\mathrm{d}}$, and $w_{\mathrm{d}}$ on the $x z$ plane at a specified depth $y$; differentiation of the interpolating functions determines the $x$ and $z$ gradients. The cycle is repeated until no change in the velocity field occurs by further repetition.

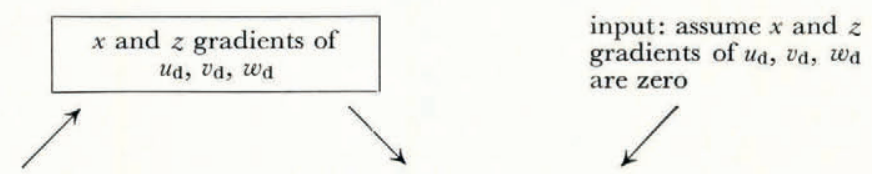

3. fit interpolating functions

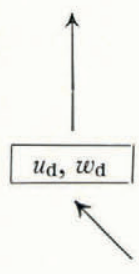

I. combine with $\partial u_{\mathrm{s}} / \partial x, \partial v_{\mathrm{s}} / \partial z$, etc. integrate $\frac{\partial v}{\partial y}=-\left(\frac{\partial u}{\partial x}+\frac{\partial w}{\partial z}\right)$

2. use bore-hole displacements

Fig. 2. Iterative cycle for calculation of velocity and strain-rate.

In proposing this scheme it is implicity assumed that the number and arrangement of bore holes is suitable for estimating both the $x$ and $z$ gradients. Clearly the minimum array is a set of three non-colinear bore-hole sites such as that used by Kamb and Shreve (I966). In such an array one is constrained to use linear interpolation between the bore holes for estimation of the $x$ and $z$ gradients. In a more extensive array of bore holes, more complicated interpolating functions are possible. The choice of form for the interpolating functions and some assessment of the reliability of the interpolation is a crucial step in the analysis.

\section{An EXAmple}

Nine holes were bored into the Athabasca Glacier in 1966. They were arranged in an approximately square grid. Grid lines extended across and along the length of the glacier and were spaced $150 \mathrm{~m}$ apart or about half the maximum depth of the glacier. The initial arrangement of the bore holes and associated surface markers is shown in Figure 3 . The absolute and relative locations of surface markers and the surface intersection of bore holes were determined by triangulation and tape measurements in r966 and again in r967 about one year later. Similarly the initial and final coordinate profiles of bore holes were determined by inclinometry surveys. The relationship of the surface markers and bore holes to the glacier geometry, the results of the measurements, and their interpretation are presented in separate papers. Here the aim is to illustrate how the above method of analysis can be applied and some of the practical problems which can arise in its application.

For this application the azimuth of the $x$ axis was chosen to be equal to that of the average ice velocity measured at the surface. In this case the $y z$ and $x y$ coordinate planes are transverse sections and vertical longitudinal planes respectively. The $x, y$, and $z$ components of velocity $u, v$, and $w$ are then the longitudinal, surface-normal and lateral components of velocity.

Following the procedure outlined in the previous section, velocity components and their gradients were calculated at the surface for each bore-hole site. This was accomplished by first using the tape measurements reduced by the method of Nye (1959) in conjunction with the triangulation measurements to determine the distribution of the $x$ and $z$ gradients of 
velocity. Displacements of stakes and bore-hole surface intersections were then interpreted in terms of velocity at their initial locations.

The value of $v_{\mathrm{b}}$ to be used in Equation (19) was calculated from Equation (18) on the basis of bed orientation determined seismically by Paterson and Savage (1963) and the initial depths of holes reaching bedrock. (All holes except hole $4 \mathrm{~A}$ reached bedrock.)

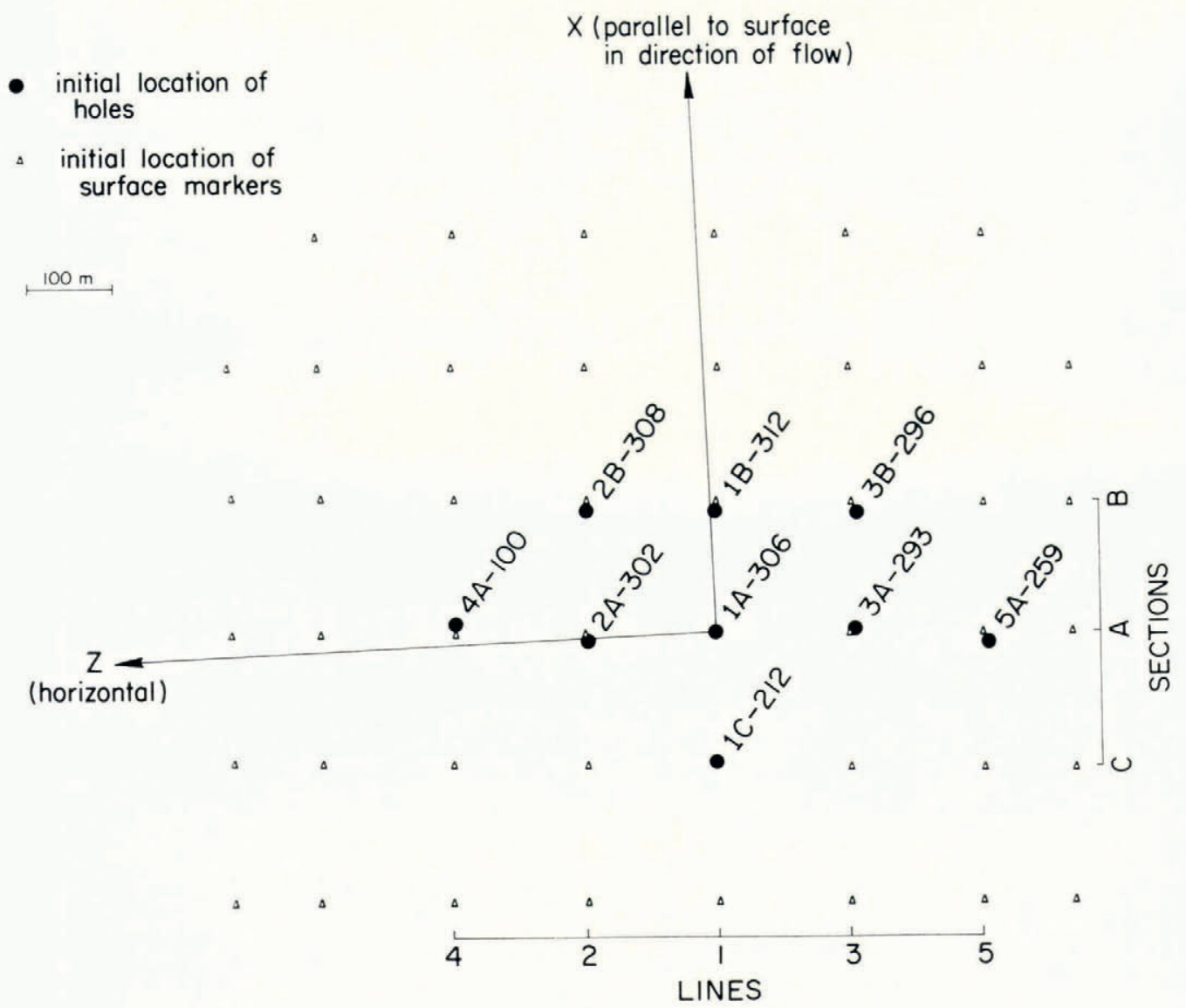

Fig. 3. Arrangement of bore holes and surface markers. Holes are named according to their location in a grid of longitudinal lines (denoted by numerals) and lateral sections (denoted by letters). Depths in meters to which deformation data were obtained is indicated for each bore hole.

In the iterative cycle (Fig. 2), it was assumed that $\partial v / \partial x=\partial v / \partial z=0$ at all depths. This eliminated the need for fitting a separate interpolating function to the calculated values of $v_{\mathrm{d}}$ at the bore-hole sites and simplified the analysis. Because of the actual smallness of the quantities at the surface and the indirect way in which they enter into the calculation of the components of velocity by Equations (I6) and (I9), this assumption produced negligible effect on the results of the calculation. (This would not necessarily be the case in a region of a glacier where the surface-slope gradients are large.)

Method of interpolation. For interpolation of $u_{\mathrm{d}}$ the following polynomial was used at depth $y$ : 


$$
\begin{aligned}
u_{\mathrm{d}}(x, y, z)=C_{0}(y)+ & C_{\mathrm{I}}(y) x+C_{2}(y) x z+C_{3}(y) z+ \\
& +\frac{1}{2} C_{4}(y) x z^{2}+\frac{1}{2} C_{5}(y) z^{2}+\frac{1}{6} C_{6}(y) z^{3}+\frac{1}{2} C_{7}(y) x^{2}+ \\
& +\frac{1}{24} C_{8}(y) z^{4}+\frac{1}{6} k_{1}(y) x z^{3}+\frac{1}{2} k_{2}(y) x z^{4} .
\end{aligned}
$$

The terms with coefficients $C_{0}$ to $C_{8}$ represent a determinate polynomial particularly suited to the arrangement of the nine bore holes (Fig. 3). The five holes of section A made it possible to estimate lateral gradients of $u_{\mathrm{d}}$ up to order 4 . The hole pairs along the lines $\mathrm{I}, 2$, and 3 made it possible to estimate the lateral gradients of $\partial u_{\mathrm{d}} / \partial x$ up to order 2. From the three holes in line $\mathrm{I}, \partial^{2} u_{\mathrm{d}} / \partial x^{2}$ could be estimated. The additional terms with coefficients $k_{\mathrm{I}}$ and $k_{2}$ were added in order to make it possible to constrain the values of $\partial u_{\mathrm{d}} / \partial x$ at holes $4 \mathrm{~A}$ and $5 \mathrm{~A}$,

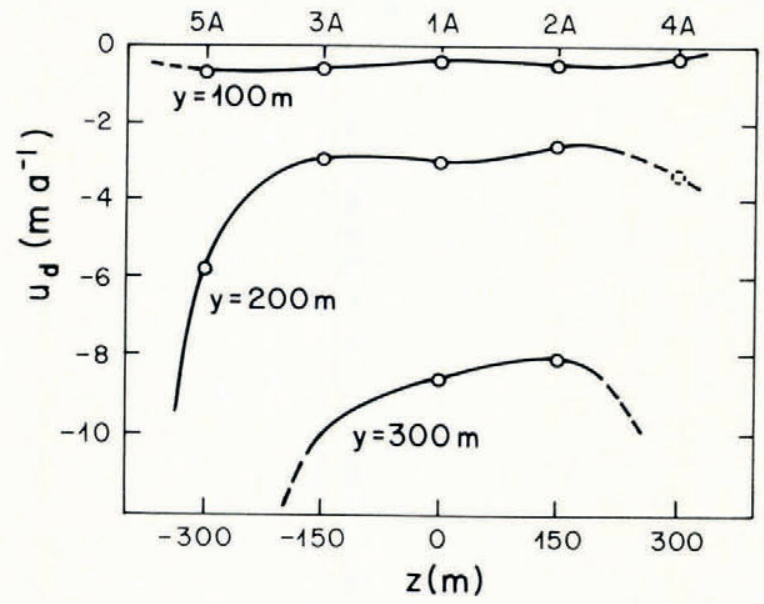

Fig. 4. $u_{\mathrm{d}}$ versus $z$ for selected depths at section $A$.

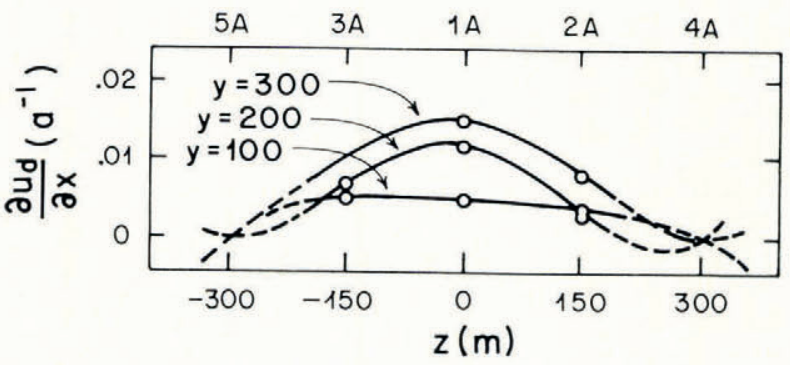

Fig. 5. $\partial u_{\mathrm{d}} / \partial x$ versus $z$ for selected depths at section $A$.

where no direct information on $\partial u_{\mathrm{d}} / \partial x$ was available and extrapolation based on a determinate polynomial with nine coefficients gave unacceptable results. For the purposes of the calculation it was assumed that $\partial u_{\mathrm{d}} / \partial x=\mathrm{O}$ at all depths in holes $4 \mathrm{~A}$ and $5 \mathrm{~A}$. (This is equivalent to assuming that $\partial u / \partial x$ is independent of depth and is equal to the value measured at the surface.) The values of $u_{\mathrm{d}}$ at the nine bore holes and the two constraints determined the eleven coefficients $C_{0}$ to $C_{8}, k_{\mathrm{I}}$ and $k_{2}$. Examples of the interpolation on the converged values of $u_{\mathrm{d}}$ at various depths are shown in Figures 4 and 5 .

In this case the data put fairly narrow constraints on the possible interpolating functions which could be reasonably fitted to them. For this reason one can have reasonable confidence 
in the calculated values of $\partial u / \partial x$ and $\partial u / \partial z$, especially near the center of the array. The only direct assumption about the velocity gradients at the bore holes is the constraint placed on $\partial u_{\mathrm{d}} / \partial x$ in holes $4 \mathrm{~A}$ and $5 \mathrm{~A}$ at the lateral margins of the array.

Interpolation on $w_{\mathrm{d}}$ could have been made in similar fashion. However, $w_{\mathrm{d}}$ does not have a consistent trend across the bore-hole array, as exists in the case of $u_{\mathrm{d}}$. Use of a polynomial equivalent to that of Equation (20) accentuated the complexity of the distribution. For this reason simple differences gave more acceptable values for the $x$ and $z$ derivatives of $w_{\mathrm{d}}$.

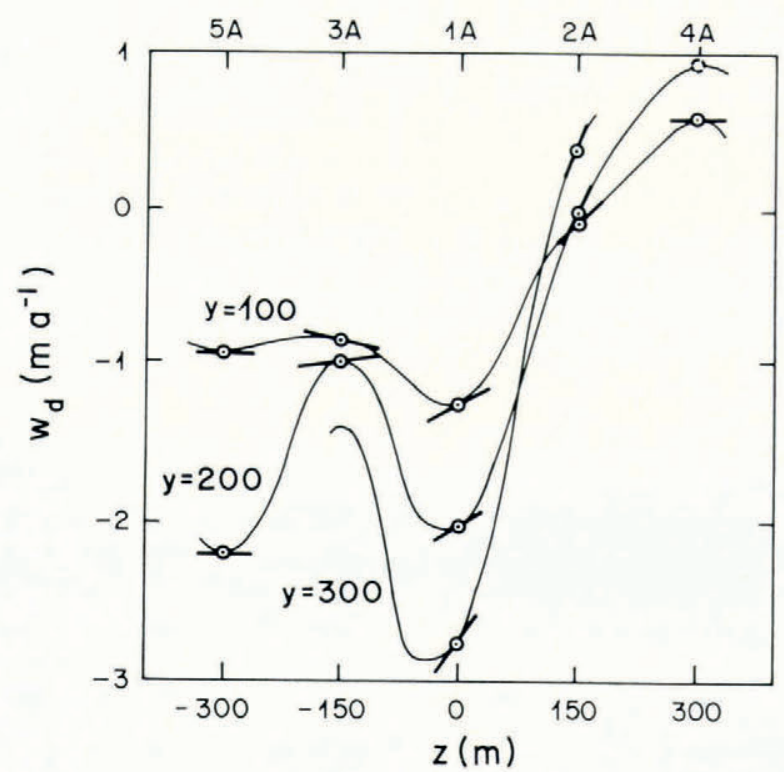

Fig. 6. $w_{\mathrm{d}}$ versus $z$ for selected depths at section A. Computed derivatives $\partial w_{\mathrm{d}} / \partial z$ are represented by line segments; curves represent interpolating functions which are compatible with the computed slopes.

Table I. Formulae for derivatives of $w_{\mathrm{d}}$

$\begin{array}{ccc}\text { Hole } & \partial w_{\mathrm{d}} / \partial x & \partial w_{\mathrm{d}} / \partial z \\ \text { IA } & \left(K_{1}+K_{9}\right) / 2 & \left(K_{3}+K_{6}\right) / 2 \\ \text { IB } & \left(3 K_{1}-K_{9}\right) / 2 & \left(K_{2}+K_{5}\right) / 2 \\ \text { IC } & \left(3 K_{9}-K_{\mathrm{I}}\right) / 2 & 0 \\ \text { 2A } & K_{4} & \left(K_{\mathrm{IO}}+K_{3}\right) / 2 \\ \text { 2B } & K_{4} & 0 \\ \text { 3A } & K_{7} & \left(K_{6}+K_{8}\right) / 2 \\ \text { 3B } & K_{7} & 0 \\ \text { 4A } & 0 & 0 \\ \text { 5A } & 0 & 0\end{array}$

This corresponds to fitting simple functions (straight lines and parabolas) locally, rather than attempting to choose an analytically expressible function applicable over the whole array. Examples of the calculated values of $\partial w_{\mathrm{d}} / \partial z$ are shown in Figure 6 . To express the specific formulae let $w_{\mathrm{d}}^{\mathrm{M}}$ represent $w_{\mathrm{d}}$ at bore hole $\mathrm{m}$ in meters per year. Then with the

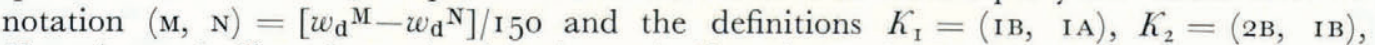
$K_{3}=(2 \mathrm{~A}, \mathrm{IA}), K_{4}=(2 \mathrm{~B}, 2 \mathrm{~A}), K_{5}=\left(\mathrm{iB}, 3^{\mathrm{B}}\right), K_{6}=\left(\mathrm{IA}, 3^{\mathrm{A}}\right), K_{7}=(3 \mathrm{~B}, 3 \mathrm{~A}), K_{8}=\left(3 \mathrm{~A}, 5^{\mathrm{A}}\right)$, $K_{9}=(\mathrm{IA}, \mathrm{IC})$, and $K_{\mathrm{I0}}=(4 \mathrm{~A}, 2 \mathrm{~A})$, the formulae for the derivatives are as in Table I.

At holes $4 \mathrm{~A}$ and $5 \mathrm{~A}$, where no direct information on $\partial w_{\mathrm{d}} / \partial x$ is available, $\partial w_{\mathrm{d}} / \partial x$ was set equal 
to zero. At holes $1 \mathrm{C}, 2 \mathrm{~B}, 3 \mathrm{~B}, 4 \mathrm{~A}$, and $5 \mathrm{~A}$, where lateral extrapolation of $w_{\mathrm{d}}$ must be completely hypothetical, $\partial w_{\mathrm{d}} / \partial z$ was set equal to zero. These assumptions are equivalent to setting the gradients of velocity in question equal to their surface values at all depths.

In contrast with the experience in interpolation of $u_{\mathrm{d}}$, the data are ambiguous in the case of $w_{\mathrm{d}}$. It is clear from Figure 6 that quite different curves could be fitted to the data. Thus the choice of a particular interpolating scheme is a subjunctive judgement involving a number of arbitrary assumptions.

Since the bore holes were not all measured to the same depth (Fig. 3), a practical problem arises in the extension of the calculations to depths greater than that of the shallowest hole (in this case, hole 4A). Below that depth the above interpolating schemes could not be applied without modification. This problem was overcome as follows. First the coordinate profile of hole 4A was extrapolated to a depth of $200 \mathrm{~m}$. Then starting from the surface, interpolation was done using all nine points down to the depth of the extrapolated profile of hole $4 \mathrm{~A}$. At this point $C_{8}$ in Equation (20) and $K_{\mathrm{IO}}$ of Table I were extrapolated to the depth of the deepest hole. Between the bottom of $4 \mathrm{~A}$ as extrapolated and the next shallowest bore hole, hole ic, the ten coefficients $C_{0}$ to $C_{7}, k_{1}$, and $k_{2}$ were determined from the extrapolated value of $C_{8}$, the values of $u_{\mathrm{d}}$ at the eight remaining holes, and the two constraints on $\partial u_{\mathrm{d}} / \partial x$. Similarly $K_{\mathrm{I}}$ to $K_{9}$ were calculated from the values of $w_{\mathrm{d}}$ at the remaining holes. At the bottom of hole Ic, $C_{7}$ and $K_{9}$ were extrapolated with a resultant reduction in the number of coefficients to be determined in Equations (2O) and (2I) at greater depth. This was continued until the bottom of the deepest hole was reached. The coefficients $C_{n}$ and $K_{n}$ were extrapolated in order of decreasing subscript. Extrapolation of each coefficient was done by fitting a parabola to the trend of the coefficient over the $80 \mathrm{~m}$ depth interval above the deepest point at which it could be calculated.

Extrapolation of the bore-hole profiles and the interpolating coefficients may seem to be an artificial creation of non-existent data. However, it is clear that limits can be placed on ice velocity immediately below the bottom of a bore hole from observations in the hole. Thus it makes no sense to ignore completely the existence of the hole in calculations at greater depth, and some improvement in accuracy of the $x$ and $z$ gradients calculated in adjacent bore holes can be expected from a reasonable extrapolation of some sort.

Convergence of the iterative cycle. The iterative cycle (Fig. 2) was programmed for application on a digital computer. For this purpose the continuous curves of the bore-hole profiles were represented in the computer by coordinate values spaced at $5 \mathrm{~m}$ intervals, with coordinates at intervening depths being computed by interpolation. Calculation of the components of velocity and their $x$ and $z$ gradients was done for depths spaced $5 \mathrm{~m}$ apart. For convergence it was required that the change in any component of velocity be less than $0.0 \mathrm{I} \mathrm{m} \mathrm{a}^{-1}$ at all points. This condition was met after five traverses of the cycle and a computation time of $50 \mathrm{~s}$ on an IBM 7094 computer. Maximum change in velocity at any point was $1.29,0.26$, $0.0 \mathrm{I}$, and less than $0.005 \mathrm{~m} \mathrm{a}^{-1}$ between the consecutive traverses of the cycle. The depth distribution of $u_{\mathrm{d}}$ at the site of hole IA computed under (I) the assumption that deformation is simple shear parallel to the surface (i.e. $v$, and $x$ and $z$ gradients of velocity are zero), under (2) the assumption that the $x$ and $z$ gradients are equal to their surface values over the whole depth (i.e. after one traverse of the cycle), and from (3) the convergent iterative cycle are shown in Figure 7. In this case near the bottom the converged result agrees very well with the simplest treatment (I) above, because the longitudinal strain-rate turns out to be essentially zero there and $v$ is small since the bed is approximately parallel to the $x$ axis. Over the whole depth range, the differences between the three treatments is not great. However, where there is greater surface slope and larger longitudinal strain-rate than exists for the Athabasca Glacier in the region of the bore holes (surface slope 4 , longitudinal strain-rate $-0.02 \mathrm{a}^{-1}$ at the surface), quite significant differences between the different treatments could exist. 
Accuracy of the calculated quantities. Errors in the values of $u$ and $w$ calculated by Equation (I6) and their $y$ gradients calculated by differentiation of Equation ( $\mathrm{I} 6$ ) are caused primarily by errors in the measurement of the bore-hole tilt and coordinate profiles. This direct source can be estimated from the known accuracy of the surface triangulation and bore-hole tilt measurements. In the Athabasca Glacier holes, standard errors for the $x$ and $z$ components of tilting rate $\left(\partial \gamma_{x} / \partial t\right.$ and $\left.\partial \gamma_{z} / \partial t\right)$ are $0.0015 \mathrm{a}^{-1}$ and $0.0019 \mathrm{a}^{-1}$ respectively. Standard errors for rate of change of the bore-hole coordinates $\left(\partial x_{\mathrm{h}} / \partial t\right.$ and $\left.\partial z_{\mathrm{h}} / \partial t\right)$ are about $0.20 \mathrm{~m} \mathrm{a}^{-1}$ for both components at the surface and respectively $0.4 \mathrm{I} \mathrm{m} \mathrm{a}^{-1}$ and $0.45 \mathrm{~m} \mathrm{a}^{-1}$ for the $x$ and $z$ components at $300 \mathrm{~m}$. Secondary sources of error come from uncertainty in $v$ and the

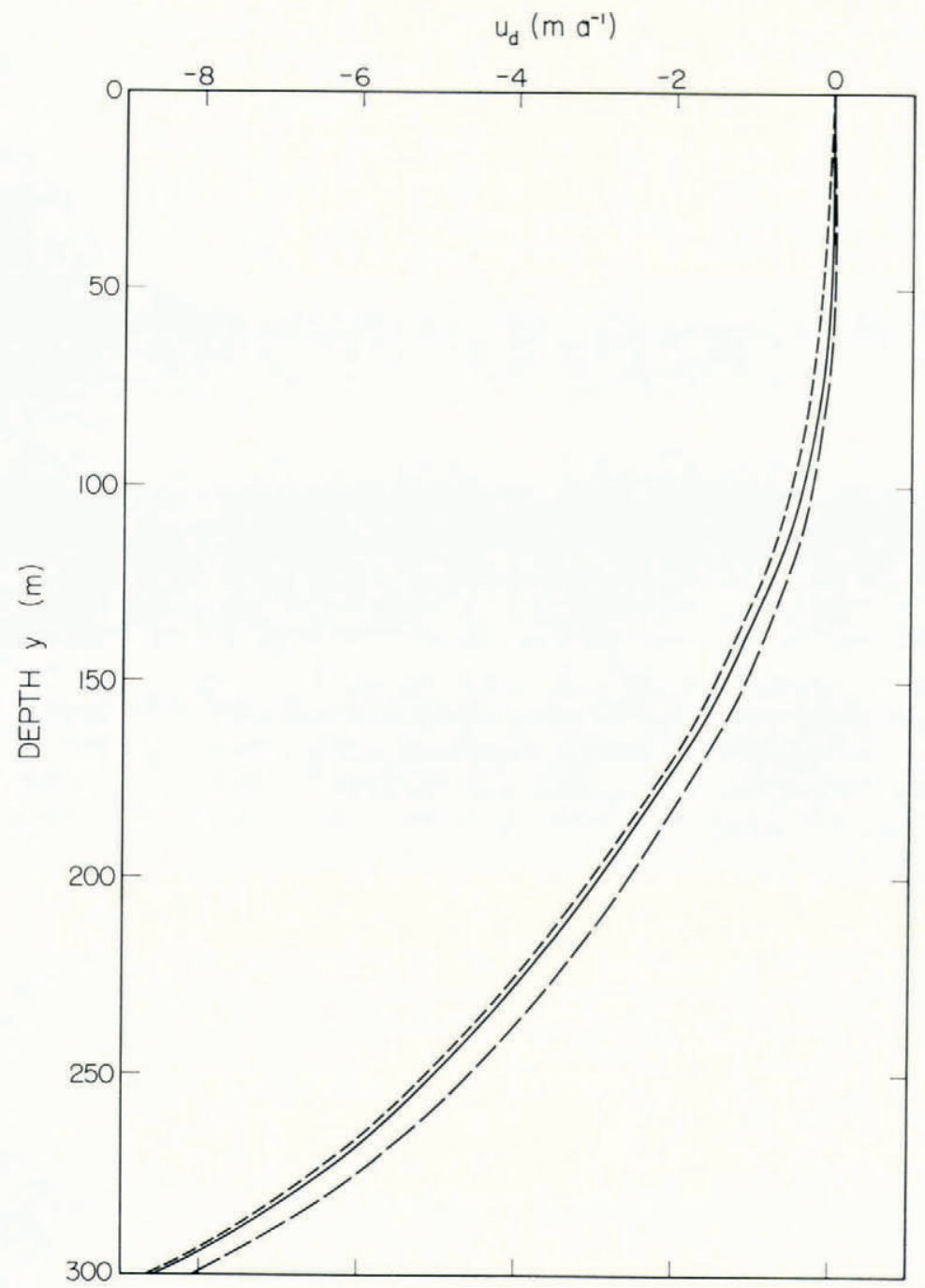

Fig. 7. Depth distribution $\mathrm{u}_{\mathrm{d}}$ computed at hole $I A$ under the assumption $(I)$ that deformation is simple shear parallel to the surface (short dashes), (2) that $x$ and $z$ gradients of velocity are independent of depth (long dashes), and from (3) the
converged iterative cycle (solid curve). 
$x$ and $z$ gradients of velocity. These are particularly difficult to evaluate, because of the subjective nature of the interpolation. Even unexpectedly large errors in these quantities produce, however, only small effect on the values calculated for $u$ and $w$ and their $y$ gradients. Maximum estimates of error for $v$ and the $x$ and $z$ gradients of velocity indicate the standard errors for $\partial u / \partial y$ and $\partial w / \partial y$ are about $0.002 \mathrm{a}^{-1}$ over the whole depth range. Similarly standard errors for $u$ and $w$ should be less than $0.22 \mathrm{~m} \mathrm{a}^{-1}$ at the surface and $0.46 \mathrm{~m} \mathrm{a}^{-1}$ at a depth of $300 \mathrm{~m}$. These values are only slightly greater than those associated with the measurement of the bore-hole profiles. Error caused by the approximate nature of Equation (I6) due to the finite time interval between measurements, and the $x$ and $z$ dependence of the $x$ and $z$ gradients of velocity turn out to be entirely negligible in comparison with the measurement errors.

Standard error in $v$ measured at the surface by triangulation is estimated to be $0.35 \mathrm{~m} \mathrm{a}^{-1}$ (except at $2 \mathrm{~B}$ where an error as large as $0.8 \mathrm{~m} \mathrm{a}^{-1}$ is possible). The value estimated at the bed from Equation ( 18 ) is judged to be accurate to within $0.5 \mathrm{~m} \mathrm{a}^{-1}$ under the assumption that bed slopes are known to within $\frac{1}{2}^{\circ}$. (At $5 \mathrm{~A}$, where the longitudinal bed slope is not known, an error as large as $0.8 \mathrm{~m} \mathrm{a}^{-1}$ is possible.) The error in $v$ over the whole depth range as calculated from Equations (I 7) and (I9) can not be calculated; however, the standard error probably does not exceed $0.5 \mathrm{~m} \mathrm{a}^{-1}$ at any depth (except for holes $2 \mathrm{~B}$ and $5 \mathrm{~A}$ ).

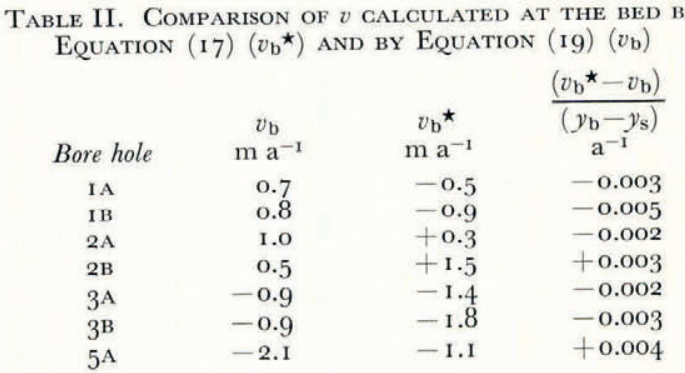

Error in the calculated values of the $x$ and $z$ gradients of velocity comes from two sources: (I) uncertainty in $u$ and $w$ at the bore-hole sites, (2) failure of the assumed interpolating functions to represent the values of $u$ and $w$ correctly between the bore-hole sites. Values estimated for the first of these range from $0.001 \mathrm{a}^{-1}$ at the surface to between 0.002 and $0.004 \mathrm{a}^{-1}$ at a depth of $300 \mathrm{~m}$ depending on whether the bore-hole site has other holes on both sides or just one side. The additional interpolation error arising from incorrect interpolation between the holes can not be quantitatively evaluated. Some information on the magnitude of this error can be obtained indirectly. Since the bore-hole array lies in the ablation zone, ice incompressibility is probably a good assumption. Thus, by comparing $v_{\mathrm{b}}$ calculated from Equation (I8) with the value at the bed obtained by Equation (17), a measure of the depth-averaged error in the sum $\partial w / \partial x+\partial w / \partial z$ is obtained. In Table II this comparison is made for those bore holes which were measured over the complete depth of the glacier. It indicates that the total error in the above sum is not any greater than would be expected from the uncertainty in velocity at the bore-hole sites and suggests that any additional error associated with the interpolation is not large.

\section{Conclusion}

Although the above method is subject to uncertainty with respect to interpolation between bore holes and the indirect calculation of $v$, it requires significantly fewer assumptions about the pattern of flow for the analysis of bore-hole data and correspondingly gives more comprehensive results. For general estimates of flow velocity at depth, the increase in accuracy of 
velocity determination afforded by the method may often not justify the effort required to apply it. However, for studies which require accurate and detailed knowledge of the threedimensional velocity field in a glacier, as is required for example in order to estimate rheological parameters and stresses within the glacier, such a method is essential.

The limitations of the method associated with the subjective nature of the interpolation between holes must exist for any method using bore holes. Indeed, the problem exists for any measurement of deformation based on tagging a finite number of discrete points or lines. It must be solved by judicious selection of the location and spacing of markers as dictated by the resolution required in the particular experiment. Beyond this basic limitation there are several limitations of the present analytical method and the existing bore-hole measurement techniques which could be modified and improved. In bore-hole experiments, where the deformation rates and strain-rate gradients are very large or the time interval very long, it could be necessary to include higher order terms in Equations ( $\mathrm{I} 6$ ) or to derive alternative formulae. A desirable modification of present methods is to tag different ice elements along the bore hole. This would make it possible to measure $v$ directly.

\section{Acknowledgements}

I am grateful to Professors Barclay Kamb and Ronald Shreve for their interest and helpful discussions. Field work and computer costs were paid for by National Science Foundation Grant GP-5447. Part of the publication cost was provided by the University of Washington under National Science Foundation Grant GU-2655.

MS. received 19 June 1970 and in revised form 27 September 1970

\section{REFERENCES}

Gerrard, J. A. F., and others. 1952. Measurement of the velocity distribution along a vertical line through a glacier, by J. A. F. Gerrard, M. F. Perutz and A. Roch. Proceedings of the Royal Society, Ser. A, Vol. 213 , No. I I I 5, p. 546-58.

Kamb, W. B., and Shreve, R. L. 1966. Results of a new method for measuring internal deformation in glaciers. American Geophysical Union. Transactions, Vol. 47, No. 1, p. 190. [Abstract.]

Nye, J. F. 1957. The distribution of stress and velocity in glaciers and ice-sheets. Proceedings of the Royal Society, Ser. A, Vol. 239, No. 12 16, p. $113-33$. Nye, J. F. 1959. A method of determining the strain-rate tensor at the surface of a glacier. Fournal of Glaciology,
Vol. 3, No. 25, p. 409-19.

Paterson, W. S. B., and Savage, J. C. 1963. Geometry and movement of the Athabasca Glacier. Fournal of Geophysical Research, Vol. 68, No. 15, p. 451 $13-20$.

Savage, J. C., and Paterson, W. S. B. ${ }_{1963}$. Borehole measurements in the Athabasca Glacier. Journal of Geophysical Research, Vol. 68, No. 15, p. $4521-36$.

Shreve, R. L., and Sharp, R. P. rg7o. Internal deformation and thermal anomalies in lower Blue Glacier, Mount Olympus, Washington, U.S.A. Journal of Glaciology, Vol. 9, No. 55, p. 65-86. 